\title{
Ageing in forensic psychiatric secure settings: The views of members of staff
}

Authors: Claudio Di Lorito, Tom Dening, \& Birgit Völlm

Affiliation: Division of Psychiatry and Applied Psychology, Institute of Mental Health, University of Nottingham

Conflict of interest: None to declare

Acknowledgements: This research was funded by the National Institute for Health Research (NIHR) Collaboration for Leadership in Applied Health Research and Care East Midlands (CLAHRC EM). The views expressed in this article are those of the author(s) and not necessarily those of the NHS, the NIHR or the Department of Health and Social Care. 


\section{Abstract}

Background. Although the prevalence of older patients in forensic psychiatric services is increasing, research around service provision for this population is very limited. We aimed to gather the views of members of staff on how well secure services are meeting the challenges of an ageing population.

Methods. Three focus groups were carried out with 13 members of staff working with older patients in secure services. A topic guide, based on the research team's previous research, guided the sessions. The focus groups were audio-recorded, transcribed and analysed through thematic analysis.

Results. Two themes were identified: (i) Identifying patients' needs, which focused on how promptly any emerging issues in the older patients are identified and reported; (ii) addressing patients' needs, which focused on how the unique needs of the older patients are addressed, once established.

Conclusions. There are unique age-related issues that may have an impact on the older patients' opportunities for recovery, including a lack of specialist training for members of staff, prolonged stay in secure care and a limited number of age-relevant activities. Far from optimal, provision requires improvement through the active involvement of the primary stakeholders.

Keywords: Older patients, forensic psychiatry, old age psychiatry, secure services, qualitative research, focus groups 


\section{Introduction}

Secure forensic psychiatric services in England and Wales offer specialist treatment to individuals, who, given the severity of their mental illness, may pose a safety risk to themselves and others.

In the United Kingdom and in several other Western countries, individuals over 50 years old in secure services represent around $20 \%$ of the patient population (Di Lorito, Dening, \& Völlm, 2018a; Di Lorito et al., 2017). This percentage is destined to grow, given the ageing of the population (Senior et al., 2013), less lenient approaches to elder crime and the more frequent prosecution of historical offences, compared to the past (RECOOP, 2015; Yorston, 2015; Moll, 2013; Frazer, 2003).

In addition, older patients experience longer stays than the overall (i.e. all ages) forensic psychiatric population (Lightbody, Gow \& Gibb, 2010), prisoners sentenced for the same offence and patients in general psychiatric settings (Völlm, Bartlett \& McDonald, 2016). These factors combined cause an accumulation of long-term and newly admitted older patients in secure care.

Despite the increasing prevalence, research around the needs and experience of older patients is very limited. A recent systematic review (Di Lorito, Völlm, \& Dening, 2018), retrieved seven studies, only one of which had a qualitative element, exploring the stakeholders' views on service provision for older patients in secure care (Yorston \& Taylor, 2009).

Given the cruciality of Patient and Public Involvement in research to inform service development / improvement (Di Lorito, Bosco, Birt \& Hassiotis, 2017), and several years 
after the qualitative study by Yorston and Taylor (2009), we deemed it timely to conduct further research in this area, by giving a voice to the main stakeholder groups in secure settings: The patients and the members of staff. The views of the patients are reported elsewhere (Di Lorito, Dening, \& Völlm, 2018b). This study aims to report the views of members of staff on how well secure services are currently meeting the challenges of an ageing population in secure care.

We were driven in our enquiry by the following research questions: (i) Are members of staff sensitive/knowledgeable about issues of ageing and flexible to accommodate the unique needs of older patients? (ii) Are arrangements in place to address the older patients' unique needs (e.g. physical, social, treatment)? (iii) Are there age-appropriate, inclusive and dedicated activities for older patients? (iv) How can the less engaged older patients (e.g. longstay patients) be encouraged to get involved in treatment / activities? (v) What can be done (if anything) to improve the current service provision to the benefit of older patients?

\section{Methods}

This study was conducted in two services within Nottinghamshire Healthcare NHS Foundation Trust: Rampton Hospital, a high secure (HS) service and the Wells Road Centre, a low secure service (LS) (Table 1). The Health Research Authority issued ethics approval for the study (REC reference: 16/EM/0505). The Research and Innovation Team of Nottinghamshire Healthcare NHS Foundation Trust granted permission to access members of staff. 


\section{Participants}

Inclusion criteria:

1. The participants have been working in either of the two services for at least one year, to have experience of secure settings.

2. They have been caring for at least one patient over 50 years old.

3. They have given full consent to take part in the study.

To maximise recruitment and ensure that the study sample was as diverse as possible, two parallel recruitment strategies were adopted: (i) Members of staff working on the wards (e.g. nurses) were recruited on-site; (ii) Members of staff not working on the wards (e.g. consultant psychiatrists) were recruited through email / professional network of the research team members. As per snowball sampling, once participants registered their interest to take part, they would refer other colleagues who may be able / willing to be involved too.

\section{Data collection}

Data were collected through focus groups. This methodology was deemed most suitable to the study aims because:

(i) More insightful data can be collected through focus groups, as participants build on each other's ideas (Leung \& Savithiri, 2009), rather than engage in individual reflection, as in one-on-one interviews.

(ii) Members of staff work in a multidisciplinary team, rather than in isolation. Focus groups allow the identification of how the team coordinates and operates to address older patients' needs in the daily practice, as they facilitate the emergence of views through participants' discussion (Kitzinger 1994, 1995). 
A member of the research team (CDL), who received training on how to conduct focus groups prior to the study, moderated the sessions. Information was collected until data saturation.

A topic guide for the focus groups was developed, based on:

- Systematic reviews around older forensic populations carried out as preliminary groundwork for the project (Di Lorito, Völlm, \& Dening, 2018).

- Findings from the patients' interviews carried out in phase one of the project (Di Lorito, Dening, \& Völlm, 2018b).

- The NICE guidelines on mental wellbeing and independence in older people (NICE, 2015)

- The primary needs to be addressed in the context of forensic care to promote patients' recovery, as outlined in the Good Lives Model for offender rehabilitation (https://www.goodlivesmodel.com/information)

- The quality indicators for recovery supporting forensic services, included in the ImROC briefing (Drennan et al., 2014)

To prevent staff responses from being shaped by researcher bias, the topic guide was only used as a loose template providing overarching themes during the sessions. In fact, the participants were invited to bring forward any topics that they might deem relevant, as long they were in line with the scope of the study.

Throughout the sessions, a number of quotations from the patients' interviews included in the companion paper (Di Lorito, Dening, \& Völlm, 2018b) were collated into a PowerPoint document and displayed, to further stimulate participants' insight into the issues that most affect patients' experience. The focus groups were audio-recorded. 


\section{Data analysis}

A member of the research team fully transcribed the sessions and transferred the transcripts onto NVivo software Version 11 (QSR International Pty Ltd., 2012). Data were analysed through an inductive approach to thematic analysis (Braun \& Clarke, 2006). Therefore, we created tentative themes and subthemes, as they emerged from the transcripts of the sessions.

All the tentative themes and subthemes were subject to expansion, restriction, or modification, until the research team agreed on a final code book (Table 2), which best reflected, categorised and synthesised the information gathered during the focus groups. The data were then extracted and coded through the codebook.

\section{Data protection}

As per the study protocol, the audio-recordings of the sessions were erased, once transcription was complete. The data were anonymised during the transcribing process. The transcripts were stored in an encrypted file in a password protected computer located in the Institute of Mental Health, Nottingham, and accessible only to the research team and the regulatory bodies, in compliance with the Data Protection Act 1998. In reporting participants' quotes in this paper, we did not provide any identifiable information, to safeguard their anonymity. The only exception was the level of security in which they worked, as we deemed this crucial to allow the reader to contextualise study findings.

\section{Results}

We carried out three separate focus groups sessions: two in HS and one in LS. Thirteen members of staff agreed to take part: Eight working in HS (four participants in each session) 
and five in LS. Several participants also had previous professional experience in medium secure units.

A wide range of professions were represented, including consultant psychiatrists $(n=2)$, specialty doctors $(n=2)$, specialist medical trainees $(n=3)$, senior mental health nurses $(n=1)$, senior practice nurses $(n=1)$, staff nurse $(n=2)$ and nursing assistant $(n=2)$. The participants worked in different directorates and units, including male mental health, male personality disorder, women, and learning disability services and all actively participated in the session. The sessions lasted an average of 42 minutes.

Through thematic analysis, we derived two main themes: Identifying patients' needs and addressing patients' needs. Each theme had several sub-themes (Table 2).

\section{Theme 1. Identifying patients' needs}

This theme explored how the participants felt in relation to the ability of members of staff and the service to identify older patients' needs.

Protocols

The participants generally felt confident of the systems in place to identify age-related physical health needs:

"I think there's good screening in place for things like prostate issues... it is on the wards" agendas to make everybody aware that there are all types of screenings being offered" (LS)

They felt that good practice in this area was driven by the growing number of older patients in secure settings: 
"In the last five years there has been a gradual recognition among forensic services that the population that they look after is going to get older" (HS)

Identification of older patients' needs was also facilitated by frequent routine health checks:

"They do these regular checks for all patients and they carry out additional health checks for elderly patients, which do help highlight their age-related needs" (LS)

Occupational Training assessment further ensured the identification of the patient's overall level of functioning:

"When a patient gets OT assessment, that actually also points out toward any issue they might have” (LS)

\section{Skills of staff}

Different members of staff exhibited different levels of confidence about their knowledge of age-related issues. The consultant psychiatrists felt that their training enabled them to promptly identify health conditions and take an appropriate course of action:

"I would say that most of us would have done some placement in elderly psychiatry as part of our training and that we feel competent to identify things like cognitive deficits" (LS)

They acknowledged, however, that the members of staff having daily contact with the patients on a daily basis such as the nurses, were better suited to determine any changes in the patients' health:

"The ward staff watch the patients every day, so they are actually much better at pointing out if there are some difficulties" (HS) 
The lack of specialist training in old age psychiatry, however, affected the extent to which the nurses felt confident in their knowledge of age-related issues:

"We would not identify what is wrong. We would just notice a change and express a concern. Because we haven't got specific training in old age” (HS)

\section{Cognitive impairment / dementia}

The participants reported several barriers to identifying cognitive impairment / dementia. The lack of specialist training and the fact that symptoms of cognitive impairment are prevalent in a range of psychiatric diagnoses might cause a delay in the identification of prodromal stages of dementia:

"There might be a delay in identifying patients with dementia, because it's not surprising for someone who has got chronic schizophrenia to have cognitive deterioration. So, staff might normalise it" (HS)

A further barrier to identify cognitive deterioration / dementia might be the unique living conditions in secure settings:

“They are in a very protective environment, cos they haven't got to do a lot of the day-to-day functions. So, I think it's harder to notice anything” (HS)

A lack of routine screening represented further impediment to the early detection of dementia:

"We are not routinely doing screens. Assessment for cognitive impairment is only led by clinical suspicion” (LS) 
Nonetheless, understanding the nature of the cognitive impairment was believed to be key to developing individualised treatment plans:

"If you do any kind of intervention beforehand, the person might have better quality of life" (HS)

Therefore, the members of staff advocated for more dementia-awareness training. This might also be promoted through dedicated policy around older forensic psychiatric patients:

"National standards for the community might not be adequate in forensic settings. National standards say that older people should be screened for cognitive impairment at 70, but perhaps we need to do that earlier. So maybe dedicated policy is needed" (HS)

\section{Theme 2. Addressing patients' needs}

This theme explored participants' views on the facilitators and barriers to addressing the unique needs of the older patients. In addition, it gathered participants' views on service improvement.

\section{Facilitators}

The participants discussed several facilitators to meet the needs of older patients.

Recognising the uniqueness of each person was essential for an individual approach to care:

"From what I've seen, staff treat patients individually and consider whatever their needs are regardless of age, because you have different needs and because you are unique in yourself"' $(H S)$ 
Sensitivity toward the issues of ageing further promoted an individualised approach to care:

"People on the ward are quite sensitive to the issue of ageing. We had this patient with mobility issues and a bespoke programme of OT had been designed for him " (LS)

Being the minority of the patients on the ward, the older patients were under the scrutiny of the members of staff:

"By and large our patients fall within the younger patients group and that maybe helps us give special consideration to the few old ones" (LS)

Older patients typically stayed in secure service for a long time. Length of stay, it was reported, might further ensure that their individual needs were best met:

"People who have reached a certain age in forensic services have usually been in the service for quite a long time and so the staff team tend to know them very well and understand their individual needs" (HS)

\section{Barriers}

Some barriers to addressing the unique needs of older patients were also discussed. A major challenge for service providers was to offer activities suitable for the older patients:

"There are a lot of walks to Derbyshire, but if your mobility is poor, how willing are you going to be to participate?" (LS)

Barrier extended to making the programme of occupational therapy engaging for patients: 
"I think there are some occupational activities here that are not tailored to the patients. Making Easter bonnets is a very childish activity and the patients might find it demeaning" $(H S)$

A programme of activities which may not be found fully engaging may expose older patients to become disillusioned about treatment, especially in the context of long-stay:

If they have been in this place for many years, and they don't get stimulated, they tend to think "this is what we've got; this is where we are" (HS)

Further barriers to recovery might be presented by the fact that, despite their prevalence and vulnerability, older patients were not recognised minority status:

"I think there is now a political expectation for services to meet the needs of minorities, but the older patients are not considered a minority" (HS)

The participants continued that, even in the presence of intensive care needs, emphasis on security would still be given priority:

"Unfortunately, risk would have the priority over care needs. We had a guy who had developed Parkinson's and he died in high security, cos he was not allowed to go into medium because of the risk. And the man could hardly walk!" (HS)

Compared to the younger patients, the older patients refrained from challenging the status quo:

“They are less likely to complain, and nobody speaks for them, so they just don't have a voice" (LS) 
Finally, the little availability of financial and staff resources left little room for service improvement:

"Across the trust, older patients are relatively isolated. If resources aren't there, you can have difficulties to say 'we are going to do something special for you'”, (HS)

\section{Service improvement}

The members of staff discussed about the potential development of dedicated secure units for older patients. They contended that the current mixed-age wards had greater benefits than disadvantages for the older patients:

"If you have people in their 50s mixing with the younger adults, it can often motivate them to keep fit and strong, keep them in touch with modern living” (HS)

Mixing issues also represented a deterrent to creating dedicated units for older patients:

“There are patients who may be suitable to this environment, but others who've just been admitted and need a completely structured treatment plan. A mix be disruptive for both groups” (HS)

In addition, the members of staff suggested that older people's units could be stigmatising:

"Most patients would not be happy about being labelled as older. You have a lot of the gentlemen here who feel they are 30 years old" (LS)

For this reason, the participants preferred the idea of specialist units based on an admission criterion of biological age (i.e. how physically well the person is/feels), rather than chronical age (i.e. how old the person is): 
"I think it should be more about ability than age. Cos the concept of age is very segregating whereas ability is more enabling. So maybe a ward dedicated to those who have chronic physical issues" (LS)

The members of staff emphasised the need to seek the active involvement of patients in improving / developing services:

"Probably, if it was tailored to their needs and if they could give an input, they would go. Maybe they don't go now because they don't see it's what they want. I think the actual point is that the question should be asked to them" (HS)

\section{Discussion}

In this study, we gathered the views of forensic psychiatric members of staff around the adequacy of secure services in addressing the unique needs of older patients. Our investigation stemmed from the need to expand the limited evidence in this area and the recognition that the input of all stakeholder groups (including staff) is crucial for service improvement. The perspective of staff proved an invaluable addition to the older patients' narratives we gathered in the companion paper (Di Lorito, Dening, \& Völlm, 2018b).

In some respects, the views of patients and staff coincided. There was consensus on the fact that, with limited resources, services struggle to offer truly tailored treatment to the older patients, who are numerically inferior to the younger patients and scattered across different wards / units. It was also agreed that another barrier to individualised treatment is the priority in secure services to rehabilitate younger offenders, potentially showing institutional ageism. 
While the patients put forward the treatability argument, contending that their (usually) consolidated patterns of mental illness and behaviour are more difficult to treat, the staff opined that the older patents are disadvantaged by their numerical minority status, which is not recognised as such. In addition, compared to younger patients, older patients may be less active help-seekers / self-advocates (Doron, 2007), and therefore, sufficient resources may not be diverted into addressing their unique needs.

The limits of the current provision are reflected in the lack of specialist training in age-related issues, which may cause a delay in the identification of conditions such as cognitive impairment / dementia, which require individualised treatment plans (i.e. dedicated occupational therapy, modification of regime / environment). Lack of dementia-awareness / training has been also reported in other restrictive settings (e.g. prisons) (Joyce \& Maschi, 2016), evidencing how, despite its prevalence and the governmental strategies in place (see: https://www.gov.uk/government/publications/challenge-on-dementia-2020-implementationplan), little has been done in forensic settings.

The members of staff also reported that, when an older patient develops a chronic, progressive or terminal condition, in considering a potential discharge to specialist services, risk and security issues might often prevail over treatment needs. The combination of illequipped services and the priority given to risk management over care needs might cause unnecessary (and unethical) prolonged stay of older patients at levels of security which are not appropriate to the person's needs, as reported elsewhere (Das, Murray, Driscoll, \& Nimmagadda, 2012).

Length of stay was indeed a recurring issue in the narratives provided by the members of staff, who agreed it had a negative mediating effect in the relationship between treatment and recovery. The model in Figure 1, developed from the narratives collected in this project, 
illustrates how prolonged stay may generate and sustain a vicious circle which makes the patient less likely to move along the care pathway (Patient A), as opposed to an acceptable length of stay, which may favour opportunities for recovery (Patient B). The two scenarios are exemplified in the two case studies illustrated in Figure 2, extrapolated from data included in the companion paper (Di Lorito, Dening, \& Völlm, 2018b).

In ensuring that secure services fulfil their statutory duty of care toward the older patients, as per Article 13 of the United Nations Principles for Older Persons, and to avoid unnecessary long-term institutionalisation, alternative strategies must be in place. In contrast with the views published in the literature (Tomar, Treasaden, \& Shah, 2005), however, the members of staff did not support the creation of wards for the older patients.

Instead, they envisioned the good potential of intensive care needs units, based on overall functioning (i.e. biological age), as opposed to (chronological) age. These could be configured as slow-stream rehabilitation units, similar to the long-stay units of European countries such as the Netherlands and Germany and could be geared for patients with intensive care needs, offering age-relevant treatment and activities in a protected and safe environment.

This study is characterised by several limitations. Although we strived to abide by the strictest parameters of qualitative study trustworthiness, which include credibility, transferability, dependability and confirmability (Lincoln \& Guba, 1985), this was not always possible. For example, we could not adopt purposive sampling, as the focus groups were carried out during working hours and only a limited number of staff were willing / able to take part. In addition, the participants worked within one single Healthcare Trust, thus making their views highly contextualised and potentially unrepresentative of the national workforce employed in secure settings. 
Although we attempted to include one medium secure service in our sample, as this is where the majority of forensic patients are found, this was not possible. The lack of some multidisciplinary team members may have reduced the representativeness of the views gathered. We wished to involve also occupational therapists, who would provide us with invaluable information around activities of daily living, and social workers, who could comment on the difficulties of community placement. However, no representatives from these professional categories registered an interest to take part.

In terms of data collection, although the topic guide and PowerPoint prompts were instrumental to stimulating insight, they may have carried moderator bias, whereby only the issues identified by the researcher might have been discussed. Some limitations were also inherent to the specific nature of focus groups (Smithson, 2000). Although it appeared that the participants were equally articulate, the views of the more outspoken, assertive and/or senior participants might be overreported. In addition, discourse around older mentally-ill offenders may have discouraged any participants with contrasting views from externalising them, and this may have reflected in only socially acceptable opinions to emerge.

Finally, having already investigated similar topics in our previous paper (Di Lorito, Dening, \& Völlm, 2018b), confirmation bias (of previous findings) might have affected data analysis. In light of these limitations, findings from this work should not be considered definitive, but should represent a platform for further research, involving more representative (i.e. national) samples and gathering in-depth narratives from staff through individual (i.e. one-on-one) interviews.

This study presents several implications for practice, policy and research. Before the debate on dedicated units for older patients is resolved, current provision may require improvement. Given the central role in the daily life of patients, members of staff should be offered 
awareness and training in age-related issues, particularly around conditions such as cognitive impairment / dementia, which are prevalent among the older patients (Di Lorito, Dening, \& Völlm, 2018b), and highly impairing for the person. Training should be accompanied by routine screenings for cognitive impairment, which contrary to other over-40s health screening (e.g. eyesight/hearing impairments, prostate, breast/gynaecological checks), are only led by clinical suspicion. Ideally, screening for cognitive impairment should be added to the annual (Care Programme Approach) CPA, to adequately identify and monitor the patient's needs.

More age-relevant activities and treatment should be offered to ensure compliance with the current policy and guidelines in favour of equality and inclusion and against age discrimination. These include the United Nations Convention on the Rights of Persons with Disabilities, the National Institute for Health and Care Excellence (NICE) guidelines on mental wellbeing and independence in ageing people (NICE, 2015) and the Care Act 2015. Across different restrictive settings, including the prison system, there are valid examples of initiatives which can inform the current (far from ideal) service provision, including peersupport programmes (Baldwin \& Leete, 2010; Moll, 2013), dedicated social events for the over 50s and modifications of environments (Hodel \& Sanchez, 2012).

In line with the members of staff involved in this study, we advocate that improvement of services requires actively seeking the involvement of patients in the debate. As evidenced in our previous study (Di Lorito, Dening, \& Völlm, 2018b), the primary stakeholders have clear views on how services can be improved. Their unique insight into ageing in secure settings would prove an invaluable contribution on impending issues, such as how the motivation of long-stay patients to keep engaged in treatment can be boosted, so that they have equal opportunities for recovery. 
Finally, service improvement may also be conditional on policy development. As reported by the staff, national standards for people in the community (e.g. the NICE guidelines on mental wellbeing and independence in ageing people) are not necessarily applicable to the unique context of secure settings and might be insufficient to ensure equal care quality across different services. Development of dedicated policy addressing the specific issues of older forensic psychiatric patients should therefore be considered. 


\section{References}

Baldwin, J., \& Leete, J. (2012). Behind bars: The challenges of an ageing population. Australian Journal of Dementia care, 1, 16-19.

Braun, V., \& Clarke, V. (2006). Using thematic analysis in psychology. Qualitative Research in Psychology, 3, 77-101.

Das, K., Murray, K., Driscoll, R., \& Nimmagadda, S. (2012). Assessment of healthcare and placement needs in an older forensic psychiatric population in comparison to a younger forensic psychiatric population: Comment. International Psychogeriatrics, 24, 1188-1190.

Di Lorito, C., Bosco, A., Birt, L., \& Hassiotis, A. (2017). Co-research with adults with intellectual disability: A systematic review. Journal of Applied Research in Intellectual Disabilities.

Di Lorito, C., Castelletti, L., Tripi, G., Gandellini, M. G., Dening, T., \& Völlm, B. (2017). The Individual Experience of Aging Patients and the Current Service Provision in the Context of Italian Forensic Psychiatry: A Case Study. Journal of forensic nursing, 13, 118125.

Di Lorito, C., Dening, T., \& Völlm, B. (2018a). The characteristics and needs of older forensic psychiatric patients: A UK cross-sectional study. Submitted for publication.

Di Lorito, C., Dening, T., \& Völlm, B. (2018b). Ageing in forensic psychiatric secure settings: the voice of older patients. The Journal of Forensic Psychiatry \& Psychology, 1-27.

Di Lorito, C., Völlm, B., \& Dening, T. (2018). Ageing patients in forensic psychiatric settings: A review of the literature. International Journal of Geriatric Psychiatry.

Doron, I. (2007). Heaven or hell? Aging behind bars in Israel. International Journal of Aging, 9, 145-159. https://doi.org/10.2190/HA.9.2.e.

Drennan, D., Wooldridge, J., Aiyegbusi, A., Alred, D., Ayres, J., Barker, R., Carr, S., Euson, S., Lomas, H., Moore, E., Stanton, D. \& Shepherd, G (2014). Implementing Recovery through Organisational Change Briefing Paper 10. London: Centre for Mental Health and Mental Health Network. Accessed 10.03.2018 at: http://www.imroc.org/wpcontent/uploads/ImROC-briefing-10-Making-Recovery-a-Reality-in-Forensic-Settings-finalfor-web.pdf

Frazer, L. (2003). Ageing inside. school for policy studies working paper series paper number 1. University of Bristol, Bristol. Retrieved from http://www.clinks.org/sites/default/files/basic/filesdownloads/Older\%20Prisoners\%20LF\%20 Report\%20sps01_lf.pdf

Hodel, B., \& Sanchez, H. G. (2012). The Special Needs Program for Inmate-Patients with Dementia (SNPID): a psychosocial program provided in the prison system. Dementia, 12, 654-660. doi:http://dx.doi.org/10.1177/1471301211432952 
Kitzinger J. (1994) 'The methodology of focus groups: the importance of interaction between research participants', Sociology of Health, 16, 103-21.

Kitzinger J. (1995) 'Introducing focus groups’, British Medical Journal, 311, 299-302

Joyce, J., \& Maschi, T. (2016). "In here, time stands still”. The rights, needs and experiences of older people in prison. Irish Penal Reform Trust: Dublin, Ireland.

Leung, F. H., \& Savithiri, R. (2009). Spotlight on focus groups. Canadian Family Physician, $55,218-219$.

Lightbody, E., Gow, R.L., \& Gibb, R. (2010). A survey of older adult patients in special secure psychiatric care in Scotland from 1998 to 2007. The Journal of Forensic Psychiatry \& Psychology, 21, 966-974. DOI: 10.1080/14789949.2010.504858

Moll, A. (2013). Losing track of time: Dementia and the ageing prison population.

London: Mental Health Foundation. Retrieved from

http://www.mentalhealth.org.uk/content/assets/PDF/publications/losingtrack-of-time-

2013.pdf?viewDStandard

National Institute for Health and Care Excellence 2015. Mental wellbeing and independence in older people. NICE guideline (NG32). London: National Institute for Health and Care Excellence.

NVivo qualitative data analysis Software (2012). Melbourne, Australia: QSR International Pty Ltd.

Resettlement and Care for Older ex-Offenders and Prisoners (RECOOP). (2015). Retrieved from http://www.recoop.org.uk/pages/resources/

Senior, J., Forsyth, K., Walsh, E., O'Hara, K., Stevenson, C., Hayes, A. et al. (2013). Health and social care services for older male adults in prison: the identification of current service provision and piloting of an assessment and care planning model. Health Services and Delivery Research 1(5). https://doi.org/10.3310/hsdr01050.

Smithson, J. (2000). Using and analysing focus groups: limitations and possibilities. International journal of social research methodology, 3, 103-119.

Tomar, R., Treasaden, I.H., \& Shah, A.K. (2005). Is there a case for a specialist forensic psychiatry service for the elderly? International Journal of Geriatric Psychiatry, 20, 51-56. DOI: $10.1002 / g p s .1247$

United Nations (1991). United Nations Principles for Older Persons. Adopted by General Assembly resolution 46/91 of 16 December 1991. Geneva, Office of the United Nations High Commissioner for Human Rights, 2013 (A/RES/46/91) (http://www.ohchr.org/EN/ProfessionalInterest/Pages/OlderPersons.aspx). Accessed 6 December 2017.

United Nations (2006). International Convention on the Rights of Persons with Disabilities. New York, NY, United Nations. 
Völlm, B., Bartlett, P., \& McDonald, R (2016). Ethical issues of long-term forensic psychiatric care. Ethics, Medicine and Public Health, 2, 36-44.

Yorston, G.A. (2015). Managing aggression and violence in older people. In G. Dickens, P. Sugarman, \& M. Picchioni (Eds.), Handbook of secure care (pp. 179-192). London: The Royal College of Psychiatrists.

Yorston, G., \& Taylor, P. (2009). Older patients in an English high security hospital: a qualitative Study of the experiences and attitudes of patients aged 60 and over and their care staff in Broadmoor Hospital. Journal of Forensic Psychiatry and Psychology, 20, 255-267. 
Table 1. Services involved in the study

Rampton Hospital

The Wells Road Centre

\begin{tabular}{r|cc} 
Level of security & High & Low \\
Foundation & 1912 & 1993 \\
Location & Retford, Nottinghamshire & Nottingham, Nottinghamshire \\
Managing Trust & Nottinghamshire Healthcare NHS Foundation Trust \\
Beds & 323 & 76 \\
Male Mental Health & $\checkmark$ & $\checkmark$ \\
Male Personality Disorder & $\checkmark$ & $\times$ \\
Women (all diagnoses) & $\checkmark$ & $\checkmark$ \\
Learning disability services & $\checkmark$ & $\checkmark$ \\
Deaf services & $\checkmark$ & $\times$
\end{tabular}


Table 2. Code book

Theme Sub-theme Explanation

\begin{tabular}{|c|c|c|}
\hline \multirow[t]{3}{*}{ Identifying patients' needs } & Protocols & $\begin{array}{l}\text { Participant discusses the protocols in place in secure service to ensure that } \\
\text { the older patients' needs are identified. }\end{array}$ \\
\hline & Skills of staff & $\begin{array}{l}\text { Participant discusses the ability of various members of staff to identify any } \\
\text { emerging health issue in the older patients. }\end{array}$ \\
\hline & Cognitive impairment / dementia & $\begin{array}{l}\text { Participant discusses how the needs of patients with cognitive impairment / } \\
\text { dementia are identified. }\end{array}$ \\
\hline \multirow[t]{3}{*}{ Addressing patients' needs } & Facilitators & Participant describes the facilitators to meet older patients' needs. \\
\hline & Barriers & Participant discusses the barriers to meet older patients' needs. \\
\hline & Service improvement & $\begin{array}{l}\text { Participant comments on how the service can be improved. Includes } \\
\text { dedicated service provision and age-relevant activities and treatment. }\end{array}$ \\
\hline
\end{tabular}


Figure 1. Mediation of long-stay in recovery outcomes

A

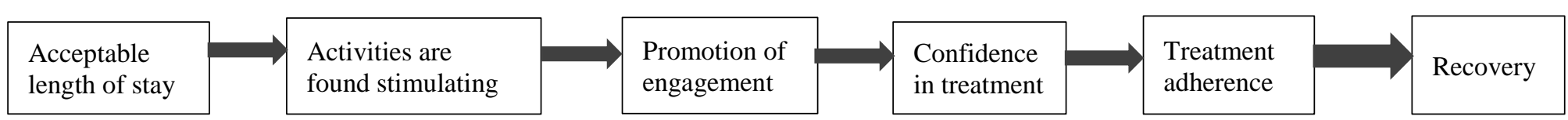

B

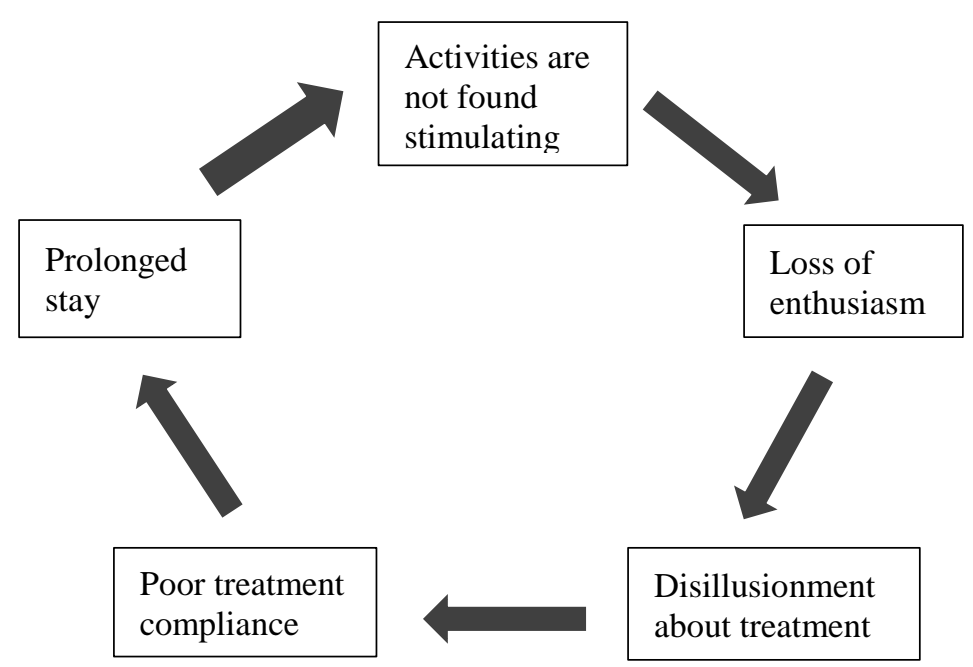


Figure 2. Scenarios of recovery and long-term institutionalisation

Patient A - Recovery

- James is a recently admitted patient in high security. He feels stimulated by the activities: "I think all of the activities that they do here, anybody can find something to do within them, so it's targeted to all ages. Take the garden for example, anybody can do some gardening"

- James firmly believes in the good of treatment: "It's about getting us prepared to move on. And it's not an easy journey, but I can see the benefits cos it's been a journey of change for me"

- He fully complies with treatment and is making good progress: "I have applied to the Ministry of Justice to get leave and the staff have supported that, all of them"
Patient B - Long-stay

- Frank is a long-term patient in high security. When asked about his daily routines, he explains: "I haven't got much to do in terms of activities, cos I've done everything. Done all the courses, done psychology"

- With time, he has become very disengaged and self-withdrawn: "I just watch television all day. There's nothing for me, so I just vegetate"

- He has lost confidence in treatment and developed resentment toward the system: "I should have never put in a position where I should fight the system. This system is in place to protect the patients"

- Because he is doing no progress, Frank is kept in high security. 